\title{
Substandard and falsified medicine screening technologies
}

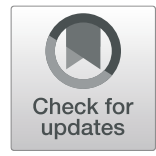

\author{
Lukas Roth $^{\dagger}$, Kevin B. Biggs ${ }^{\dagger}$ and Daniel K. Bempong ${ }^{*}$ (])
}

\begin{abstract}
Substandard and falsified medicine screening technologies are invaluable tools for post-marketing surveillance of medicine quality, particularly in low- and middle-income countries. National Regulatory Authorities and their partners leverage screening technologies to detect substandard and falsified medicines in the field and eliminate them from the medical product supply chain. An arsenal of different screening technologies has been developed to evaluate a variety of medicine quality attributes and performance characteristics, applying visual, physical and chemical analyses. Selecting the most effective screening technologies for a given quality challenge requires consideration of several practical, technical, and scientific factors, such as the type of medicines screened, the requirements of the screening technology, and the type of information needed. More widespread adoption of screening technologies will be facilitated by improved workforce development, technological advancements, and the development of a more robust supporting regulatory framework.
\end{abstract}

Keywords: Screening technologies, Substandard and falsified medicines, Post-marketing surveillance

\section{Introduction}

The proliferation of substandard and falsified (SF) medicines is a significant global concern, particularly in lowand middle- income countries (LMICs) where supply chain security is limited (Gostin and Buckley 2013; WHO 2017c). A lack of resources allocated to routine quality control (QC) amplifies the challenge of combating these products in LMICs as compared to high-income countries. The World Health Organization (WHO) estimates 10.5\% of medicines are SF (WHO 2017c). SF medicines waste resources and are linked to increases in treatment failure, adverse reactions, antimicrobial resistance, and diminished confidence in the health system (Johnston and Holt 2014; Kaur et al. 2016; Nayyar et al. 2015; Safe, Secure, and Controlled: Managing the Supply Chain of Antimicrobials 2015; WHO 2017b).

Substandard medicines are authentic products that fail to meet either their quality standards or specifications whereas falsified medicines are inauthentic products that deliberately misrepresent their identity, composition, or source (WHO 2017b). Substandard medicines are often

\footnotetext{
* Correspondence: dkb@usp.org

${ }^{\dagger}$ Lukas Roth and Kevin B. Biggs are co-first authors.

U.S. Pharmacopeia, Rockville, Maryland, USA
}

a consequence of manufacturing errors, inadequate storage, or poor distribution practices. Falsified medicines are generally produced deliberately for economic exploitation. It should be noted it may not be apparent from analytical results alone whether the determined quality deviations of an SF medicine were manufactured deliberately or arose from errors or neglect. The drug substance (or active pharmaceutical ingredient) of a substandard or falsified medicine may be absent, present in insufficient amounts, or substituted with a different drug substance. Sophisticated falsified medicines may incorporate cutting agents to dilute the strength of the claimed drug substance using a different chemical of related structure. The most blatant falsified medicines conceal their identity using the authentic packaging of the targeted product.

Screening technologies (STs) are used to rapidly detect SF medicines in the field and employ significantly less resources than those of traditional QC confirmatory technologies. The equipment associated with ST can be described as "portable", able to be transported by vehicle, or "handheld", able to be held by hand. A given ST may leverage a number of tools to help an analyst make a determination. A complete description of an ST will include the analysis type(s), principle study(s) (e.g., spectroscopy), 
technique(s) (e.g., Raman), method(s) [e.g., sample preparation schemes, identification (ID) metric, and acceptance criteria], and equipment (e.g., $785 \mathrm{~nm}$ diode laser with dispersive spectrometer). Although the operation of ST equipment is simple, effective application, method development and validation, and deployment within a regulatory system and the broader medical product supply chain requires consideration of several factors.

Employing STs is a critical component of the broader post-marketing surveillance (PMS) strategies implemented by National Regulatory Authorities (NRAs) and their partners to monitor the quality of medicines (Newton et al. 2018; Nkansah et al. 2017; Roth et al. 2018b). When applied successfully, STs significantly increase the number of samples that can be tested and reduce the number of samples requiring more resource intensive confirmatory testing at the National Quality Control Laboratory (Hajjou et al. 2013). Successful implementation of STs has benefited from collaborative efforts between NRAs, donors, and technical partners, e.g., the Asian Development Bank, the United Nations Development Programme, the U.S. Agency for International Development, the Lao Oxford Mahosot Wellcome Trust Research Unit, the U.S. Pharmacopeia (USP), and the WHO (Newton et al. 2018; PQM 2017; WHO 2015; WHO 2017a).

\section{Analysis types}

There are three ST analysis types: visual, physical, and chemical, listed here in order of increasing sophistication. Visual analysis may include inspection of packaging integrity, labeling, dosage units, and or various product security features e.g., holograms and microprinting. Physical analysis may involve the evaluation of the disintegration and or dissolution performance and may also employ microscopy, refractometry, and or refractive index measurements. Chemical analysis may include the application of, for example, spectroscopy, spectrometry, chromatography, and or wet chemistry. Although employing all three analysis types improves the confidence of detecting an SF medicine, chemical analysis offers the most direct supporting evidence. However, compared to the other analysis types, chemical analysis, with the exception of wet chemistry, often requires more consideration by the analyst to implement effectively.

\section{Method development and validation considerations}

Like any analytical method, screening methods for SF medicines, and particularly those that employ chemical analysis, benefit from a rigorous method development and validation strategy. To expand on the importance of method development and validation in screening, a comprehensive description of this process is described in Fig. 1. When the performance of a screening ID method is fully evaluated, through a comprehensive validation process, accurate conclusions can be drawn from the data.

\section{Notable techniques for substandard and falsified medicine detection Vibrational spectroscopy}

Vibrational spectroscopy encompasses three different techniques; mid-infrared (IR), near-IR (NIR) and Raman spectroscopy, each of which probe the vibrational structure of samples. Vibrational spectra are plots of photon intensity versus energy, with peak positions indicative of chemical bond energies. Each technique provides complementary information and thus can be used in combination to improve screening performance. A number of considerations should be taken into account when selecting the most effective technique, such as interferences, composition ranges, and relative component response. Vibrational spectra may also provide quantitative information with limits of detection reported as low as 1-3\% (Kauffman et al. 2010; Kovacs et al. 2014). All techniques are non-destructive and minimally invasive, enabling rapid analysis of products as-is. Vibrational spectroscopic instruments can be rapidly calibrated using reference materials, enabling the transfer of data and related ID methods between instruments. Such instruments are often outfitted with onboard data analysis capabilities, enabling the analyst to make objective ID decisions. The entire process of sample preparation, data collection, and data analysis can take less than $1 \mathrm{~min}$. Figure 2 provides an expanded discussion of vibrational spectroscopy data analysis and the application of chemometrics.

Infrared spectroscopy IR instruments measure the absorption of IR radiation. IR spectroscopy is most sensitive to polar bonds, lending this technique most responsive to functional groups. All IR instruments employ diamond attenuated total reflection (ATR) due to its robustness and ease-of-use. By pressing the sample material against the diamond ATR element (note: liquid samples may simply rest on the ATR element), the IR probe beam penetrates the surface $\sim 1-5 \mu \mathrm{m}$. This low penetration depth enables as-is sample analysis without the requirement for sample dilution as is the case for other IR techniques. The low penetration depth of IR instrumentation inhibits through package (coatings, capsules, or blister packs) analysis. In general, tablets and capsule dosage forms must be transformed into fine powders to generate reproducible measurements. Powders are more suitable for IR analysis as rigid samples introduce variations in the amount of pressure that can be applied across the ATR element surface, creating signal intensity variations. The very strong IR absorption of water may also interfere with IR data analysis. 
Screening ID methods employ hypothesis testing. The ID hypothesis for ST methods is "the material is a quality medicine (i.e., not SF)". To confirm or reject the ID hypothesis, an ID metric (e.g., peak position) is developed to produce an $I D$ value (e.g., peak position deviation [from reference]) which reflects the dissimilarity ${ }^{1}$ between test material and reference data. As no two real data sets can be identical i.e., express no dissimilarity, an ID threshold must be established to make an ID decision. For screening methods, in contrast to confirmatory ID methods (e.g., QC laboratory tests), an ID decision may be conclusive or inconclusive. A conclusive ID decision may accept or reject the ID hypothesis leading to a "positive" or "negative" ID result, respectively. An inconclusive ID decision follows from the generation of an ambiguous ID value. An ambiguous ID value is a value for which neither a positive or negative decision has been demonstrated to produce the desired level of confidence, i.e. accuracy, during method validation.

The accuracy, or diagnostic accuracy, of a screening ID method is described by the demonstrated sensitivity and selectivity of the method during method validation. ${ }^{2}$ Sensitivity and selectivity are dependent on the quality of data collected/processed, discriminating power of the ID metric, and structuring of the ID threshold. Any conclusive ID decision proven to be inaccurate - through confirmatory means - is termed a "false" ID outcome and, conversely, a "true" ID outcome if proven accurate. The accuracy of a screening method is calculated by determining the probability of producing a "false-negative" or "false-positive" outcome, termed type I $\alpha$ and type II $\beta$ errors, respectively. Method sensitivity and selectivity for SF medicine screening methods refer to the probability the method will accurately detect a quality and SF medicine, respectively, and is expressed as:

$$
\text { Sensitivity }=(1-\alpha) 100 \% \text { and Selectivity }=(1-\beta) 100 \%
$$

Once the data collection/processing and ID metric have been established, the level of sensitivity and selectivity can be optimized through appropriate structuring of the ID threshold. If the desired level of confidence cannot be achieved using a single ID threshold, a two ID threshold scheme may be employed as depicted below. A two ID threshold scheme can serve to balance the risk of making false ID decisions and be leveraged by the analyst/regulator to initiate a different $I D$ response e.g., application of an alternative ST or confirmatory test. Achieving the desired level of sensitivity and selectivity for detection of SF medicines using a single ID threshold system may be particularly challenging considering the large dynamic range of ID values that could exist. Because public safety is the priority in PMS application, a false-positive outcome poses a greater risk than a false-negative, as a false-positive outcome could result in the distribution of an unsafe medicine. An effective two ID threshold scheme for a ST application may therefore employ a positive ID threshold that reflects a higher degree of confidence in the decision compared to the negative ID threshold.



Fig. 1 Screening identification method validation (A more detailed description of screening ID methods can be in Chemical Identification and its Quality Assurance (Milman 2011))

Near infrared spectroscopy NIR instruments measure the absorption of NIR radiation diffusely reflected from samples. All NIR instruments employ a $180^{\circ}$ data collection orientation with contact generally occurring between the sample and device window (typically made of sapphire). NIR radiation probes the combination and overtone vibrations of the fundamental modes probed by IR and, as a result, NIR signal is much weaker and less resolved compared to IR. The NIR penetration depth for solid dosage form medicines can extend $\sim 1-5 \mathrm{~mm}$, enabling a more representative bulk evaluation of the medicine composition than IR or Raman. The larger penetration depth and lower absorption of NIR radiation compared to IR also enables through-package analysis. Unlike IR and Raman, the signal measured by NIR instrumentation is strongly dependent on the particle size and packing density of the sample. Accounting for sample preparation variations may require extensive preprocessing for raw NIR data. Compared to IR and Raman, NIR spectra often lack distinctive features and to compensate for this inherent lack of selectivity, a modeled based ID algorithm is often required to achieve the desired level of method performance (Neospectra 2019; Shimadzu 2019).

Raman spectroscopy Raman instruments measure the light scattered inelastically from excited chemical species. Complementing the chemical information provided by IR and NIR, Raman signal is most intense for nonpolar bonds. The preference of Raman emission from nonpolar bonds enables analysis in the presence of water, unlike IR and NIR. Raman instruments generally collect spectra in a 
Data analysis for vibrational spectroscopy generally involves the application of chemometrics, which includes multivariate data preprocessing followed by use of a multivariate ID metric, referred to here as an ID algorithm ${ }^{1}$. Successful data preprocessing minimizes extraneous signals such as noise and baseline artifacts, while preserving analyte specific responses. A common multivariate data preprocessing scheme for collected spectra involves background or "blank" spectrum subtraction followed by a Standard Normal Variate correction and a $1^{\text {st }}$ or $2^{\text {nd }}$ order Savitsky-Golay derivative filter (Guillemain et al. 2017; Tsujikawa et al. 2014b; Zontov et al. 2016). Extraneous signal variations related to real/authentic sample variability and/or data collection variability should also be accounted for prior to analysis. Data collection variability is often accounted for by including replicate spectra of quality products, typically three. By updating ID methods regularly to account for quality product variation over time, such methods can produce effective results over the lifetime of the instrument.

Vibrational spectroscopic ID algorithms may or may not be "model" based. Non-model-based ID algorithms consider the "distance" between spectra in order to identify outliers, i.e., SF medicines. Common non-model-based ID algorithms for PMS are the Correlation Coefficient, Mahalanobis distance, and $k$-nearest neighbor $(k$-NN) classification (Guirguis et al. 2017; Sacre et al. 2011; Tsujikawa et al. 2014a; Wilson et al. 2017). A more sophisticated algorithmic approach to ID is model-based. A modelbased ID algorithm relies on the determination (i.e., modeling) of latent variables, computed by a "useful" mathematical combination of the variables. An effective model based ID algorithm for PMS is Soft Independent Modeling of Class Analogy or SIMCA (Sacre et al. 2011; Zontov et al. 2016). Model-based ID algorithms may significantly improve diagnostic accuracy, especially when the inherent selectivity of the quality product spectrum is limited, i.e., composed of a limited number of ill-resolved peaks, typical of NIR spectra.

Fig. 2 Vibrational spectroscopy data analysis and application of chemometrics

stand-off, $180^{\circ}$ backscattering configuration, enabling rapid noncontact sample analysis which is not possible with IR and more challenging with NIR. Raman analysis is most effective when sample fluorescence is minimal, as the fluorescence process can interfere significantly, resulting in a broad elevated baseline that can mask the Raman signal. To minimize potential fluorescence, Raman instruments are equipped with NIR excitation sources, e.g., lasers with wavelengths at 785 or $1064 \mathrm{~nm}$. Raman instruments, like NIR and unlike IR, can collect data through visibly translucent packaging. Raman is considered a "non-background" technique as spectra are not background subtracted (although subtraction of a blank spectrum may be employed to reduce stray light). The non-background capability of Raman data collection is particularly beneficial when the sample does not completely consume the probe beam. Consuming the probe beam is not generally an issue with IR equipment as the spot size is quite small: however, in the case of NIR equipment, a white or black background material is often required when the probe beam is not completely consumed by the sample (Table 1 ).

\section{$X$-ray fluorescence spectroscopy}

$\mathrm{X}$-ray fluorescence (XRF) spectroscopy employs high energy X-rays to generate fluorescence representative of the inner electronic energy levels of atomic species. The fluorescent emission from medicines can be used to determine their elemental composition (Bruker 2016; Rodriguez et al. 2014). In some cases, XRF spectra may

Table 1 Summary of vibrational screening technologies

\begin{tabular}{llll}
\hline Characteristic & Infrared & Near Infrared & Raman \\
\hline Principle & Fundamental absorptions & Overtone and combination absorptions & Emissions \\
Chemical sensitivity & Polar bonds & Polar bonds & Nonpolar bonds \\
Inherent selectivity & High & Low (modeling often required) & High \\
Sampling mode & Diamond ATR & Diffuse reflectance & $180^{\circ}$ backscattering \\
Sampling configuration & Intimate contact with sample required & Stand-off detection possible but can & Stand-off \\
& & be challenging & Monochromatic NIR i.e., \\
Excitation source & Broadband IR & Broadband IR & 785 and 1064 nm \\
Interferences & Water and any packaging & Water, particle size/packing variability & Fluorescence and opaque \\
& & packaging & $\sim 1 \mathrm{mg}$ \\
Minimum sample size required & At least $1 \mathrm{mg}$ & $\sim 20-50 \mathrm{mg}$ & $\sim 10-15 \mu \mathrm{m}$
\end{tabular}

Content from table 1 is pulled from the following references - Kauffman et al. (2010), Neospectra (2019), Rodriguez et al. (2014), Shimadzu (2019) and Zou et al. (2018) 
be used to identify coating agents and certain excipients near the surface of a medicine. The sensitivity of XRF methods increase as the atomic number of the analyte increases with heavy metals exhibiting a particularly strong response (Bortoleto et al. 2005; Kauffman et al. 2007). Both portable and handheld XRF spectrometers are commercially available. Analysis times are generally under $1 \mathrm{~min}$ (Zou et al. 2018). XRF is non-destructive and although both $\mathrm{X}$-rays and gamma rays can be used to generate XRF spectra, for user safety, portable instruments typically limit excitation to X-rays (Rodriguez et al. 2014). Field use often centers on ID applications although semi-quantitative and quantitative analysis is possible (Bruker 2017). Two commercially available handheld XRFs (the Thermo Scientific Niton XL3t and the Shimadzu EDX 700) have been used in the field to detect falsified medicines that target three combination antiretroviral tablets. (Ortiz et al. 2012; WHO 2013). Due to weak XRF response from organic material, the ID of many drug substances is challenging (Ortiz et al. 2012; Rodriguez et al. 2014).

\section{$X$-ray diffraction spectroscopy}

X-ray diffraction (XRD) spectroscopy evaluates crystal structures. By measuring the intensity patterns of scattered monochromatic X-rays, the interatomic spacing between chemical bonds can be determined (Kohli and Mittal 2012). These crystal structures can identify drug substances and their various polymorphs as well as medicine coatings and excipient profiles (Gostin and Buckley 2013; Martino et al. 2010; Zou et al. 2018). The only XRD spectrometer commercially available, is the Terra Portable (Olympus Corp). XRD techniques can identify the drug substance but, because of interference from coatings and surface roughness, successful ID generally requires homogenizing the sample (Martino et al. 2010). XRD spectroscopy methods lack sensitivity and is not quantitative (Maurin et al. 2007). Because of the poor performance of this technique and its limited availability, XRD is seldom used for the detection of SF medicines.

\section{Ion mobility spectrometry}

Ion mobility spectrometry (IMS) is a separation method used to identify volatile and semi-volatile organic compounds based on differences in gas phase ion mobility. Ion mobility is facilitated by a voltage gradient through a drift tube with detection provided by a Faraday plate. Ion mobility is dependent on the ion size, shape, and charge (Rodriguez 2017; Rodriguez et al. 2014). An internal calibrant is used to correct for day-to-day and instrument-to-instrument variations (Rodriguez et al. 2014). Because of high proton affinities, amines, a common functional group in many drug substances, provide a strong IMS response and detection limits have been demonstrated at the nanogram level (Kauffman et al. 2010; Rodriguez et al. 2014).

Although both portable and handheld IMS instruments are commercially available, most studies have been performed with portable rather than handheld instruments. Portable instruments have been used to identify amine-containing drug substances, such as sibutramine, and several erectile dysfunction drug substances in dietary supplements (Gryniewicz et al. 2009; Rodriguez et al. 2014; Safe, Secure, and Controlled: Managing the Supply Chain of Antimicrobials 2015). Like other separation techniques, such as chromatography, IMS sample preparation requires an extraction step. Furthermore, current instruments are limited to identifying small volatile molecules (Rodriguez 2017).

\section{Notable equipment for substandard and falsified medicine detection \\ Counterfeit detection device: employing multi-spectral imaging}

The Counterfeit Detection Device (CDx) was originally developed by the U.S. Food and Drug Administration (FDA). CD5 (Version 5) is a portable multi-spectral imaging tool for analyzing a wide variety of materials but will focus on the detection of SF medicines (Ranieri 2018). The CD5 uses high-intensity light emitting diodes (LEDs) of ultraviolet-visible (UV) and IR wavelengths to excite samples. The system combines a digital camera and multiple detection filters with this combination of light sources to reveal sample characteristics which are not visible under normal viewing conditions. The CD5 leverages differences in the fluorescence arising from the coating and packaging of SF medicines when compared with reference samples. The CD5 utilizes a 7-in. touch screen to display images of test samples and reference samples side-by-side for easy comparison and can store libraries of images. A digital microscope is incorporated in some configurations to examine medicine morphology and the details of package printing (Ranieri et al. 2014). The first prototypes were delivered for initial evaluation by the U.S. FDA in mid-2018. The device is expected to be commercially available as early as the end of 2019 and is currently being enhanced based on user feedback.

\section{Counterfeit drug identifier: employing multi-wavelength absorption}

The counterfeit drug identifier (CoDI) is a handheld instrument that measures the amount of light passing through a tablet exposed to a laser. The intensity and wavelength of the transmitted light results in a unique value, which depends on the physicochemical characteristics of the sample (i.e., thickness, density, color and 
chemical composition of particular brands of medicine). A falsified or degraded medicine can be quickly identified if the values are outside a specified range. Analysis is also non-destructive. Although prototypes are being evaluated, the instrument is not yet commercially available (Green 2018; Green et al. 2015).

\section{GPHF-Minilab $^{\mathrm{TM}}$ : employing chromatography and \\ disintegration}

The GPHF-Minilab ${ }^{\text {тм }}$ or, as it is commonly known, Minilab, is a laboratory kit developed by the Global Pharma Health Fund (GPHF). Minilab can analyze 90 different drug substances in multiple dosage forms and GPHF has developed approximately five new methods each year since the kit's inception in 2008. Minilab can be used for chemical and physical analysis, employing thin layer chromatography (TLC) and disintegration techniques, respectively. Minilab has been used extensively for PMS around the world and has been supplied to 97 countries (Batson et al. 2016; Fadeyi et al. 2015; GPHF 2019; Kaale et al. 2016; Lalani et al. 2015; Petersen et al. 2017). TLC is a semi-quantitative technique that separates components in a sample according to differences in polarity, as the components interact between mobile and stationary phases. Quantification of TLC data is typically performed by visually comparing the size and intensity of the spot arising from the sample solution eluted drug substance with that of the external standard solution drug substance spot of an external standard solution prepared at specific percentages of the nominal concentration. While TLC can be employed for confirmatory testing, the Minilab tests are not as accurate, precise, or sensitive as confirmatory testing and do not comply with compendial standards. Minilab methods typically use reference standard solutions at $80 \%$ and $100 \%$ of the nominal concentration but these percentages can be changed depending on user requirements (see Fig. 3). Confidence in the quantification of TLC data may be compromised if spots are large or diffuse, or if significant tailing is present. An estimate of the number of impurities is obtained by comparing the number of spots in the sample with the number of spots in the standard.

The performance of Minilab methods have produced mixed results. Minilab TLC analysis has demonstrated insufficient sensitivity in some cases to detect impurities when present at levels above specification limits (Fadeyi et al. 2015; Jaehnke 2008; Jaehnke 2017; Sabartova et al. 2011). Another study showed Minilab lacked sufficient selectivity as it was unable to distinguish between sulfadoxine and sulfamethoxazole (Khuluza et al. 2016). A recent study looking at the identity and composition of various antimalarials found sufficient sensitivity was achieved using the Minilab TLC method as it correctly detected 11 of the 14 substandard medicines. The remaining three substandard medicines were missed because of the semi-quantitative limit of the method and operator error (Batson et al. 2016). Minilab has been shown to successfully detect falsified medicines containing incorrect drug substances (IDDO 2018; Khuluza et al. 2016; Vickers et al. 2018). Disintegration time is an indirect measure of drug substance release that can be leveraged to detect SF medicines. While the Minilab

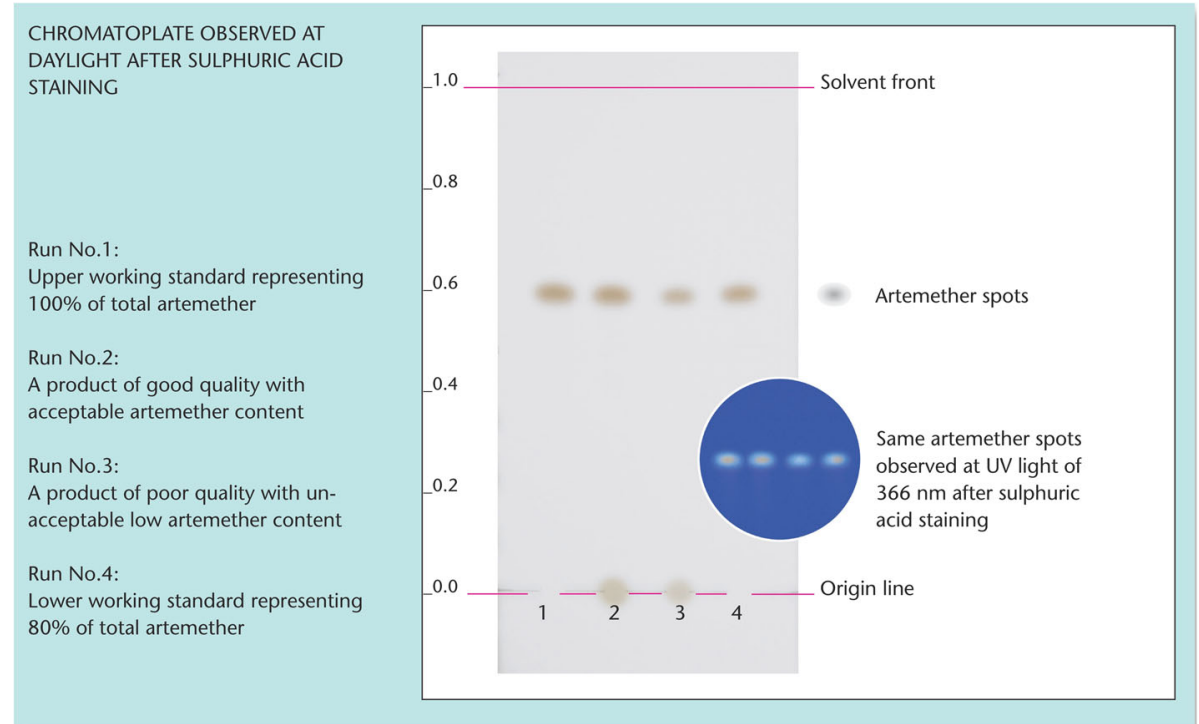

Fig. 3 Mock TLC plate of artemether from Minilab manual. Source: Global Pharma Health Fund, permission granted by Richard Jaehnke, Global Pharma Health Fund 
disintegration method has been shown to detect substandard drug substance release, such methods often lack the selectivity of dissolution testing (Sabartova et al. 2011).

System suitability is not required for Minilab. Various reagents, solvents, and consumables are required to use Minilab. One kit contains enough supplies to analyze approximately 1000 test samples. Reagents, consumables, and secondary reference standards are included in a protective case, which weighs approximately $25 \mathrm{~kg}$. Solvents are not in the protective case and weigh approximately $25 \mathrm{~kg}$ (GPHF 2017). The shelf life of the secondary reference tablets is generally 2 years. Most solvents have five-year shelf lives if stored in their original packaging. Minilab analysis types, both TLC and simple disintegration, are destructive. Analysis of a sample generally takes between one to $3 \mathrm{~h}$ depending on the proficiency of the user, but samples can be collected in parallel using multiple plates (Risha et al. 2008). To reduce operator error and improve accuracy, a cradle to hold TLC plates, which interfaces with a smartphone application that uses a quantitative algorithm to analyze spots, is being developed (Yu et al. 2016).

\section{Paper analytical device: employing chromatography and wet chemistry}

The Paper Analytical Device (PAD) was developed at the University of Notre Dame (Indiana, USA). Twelve lanes on a paper card contain reagents, which react with specific chemical functional groups to produce a color reaction. (Lieberman 2017). The sample is applied in a line across the lanes and the card is placed on its edge in water. The water is drawn up the card by capillary action and mixes the reagent with a sample to start 12 color reactions. One lane acts as "timer" to indicate when the card is to be removed from the water. The colors produced and their position within the lanes serve as a "bar code" indicative of functional groups present. This pattern can then be compared to "bar codes" of reference medicines (Weaver and Lieberman 2015; Weaver et al. 2013). Currently, the PAD can analyze over 60 drug substances. Although the current 12 lane PAD is qualitative, an additional quantitative test card, which aims to quantify ampicillin and amoxicillin and 12 additional antibiotics, is in development (Lieberman 2017)

No calibration, verification, or system suitability is required prior to using a PAD. Apart from water, no consumables or reagents are needed to perform an analysis. Sample preparation takes less than 5 min while a run takes 3 minutes. Colors develop in a further 3 minutes (see Fig. 4) and the test result can be recorded by photographing the PAD. Experienced users can interpret a PAD in less than a minute. The developers of the PAD are also in the process of developing an application to run an image analysis program that automatically analyzes the PAD, removing user subjectivity (Bannerjee et al. 2016). Individual test cards are single-use, cost USD $\$ 1$, are seven by $11 \mathrm{~cm}$ in size, and can be disposed of along with other non-hazardous waste (Lieberman 2017).

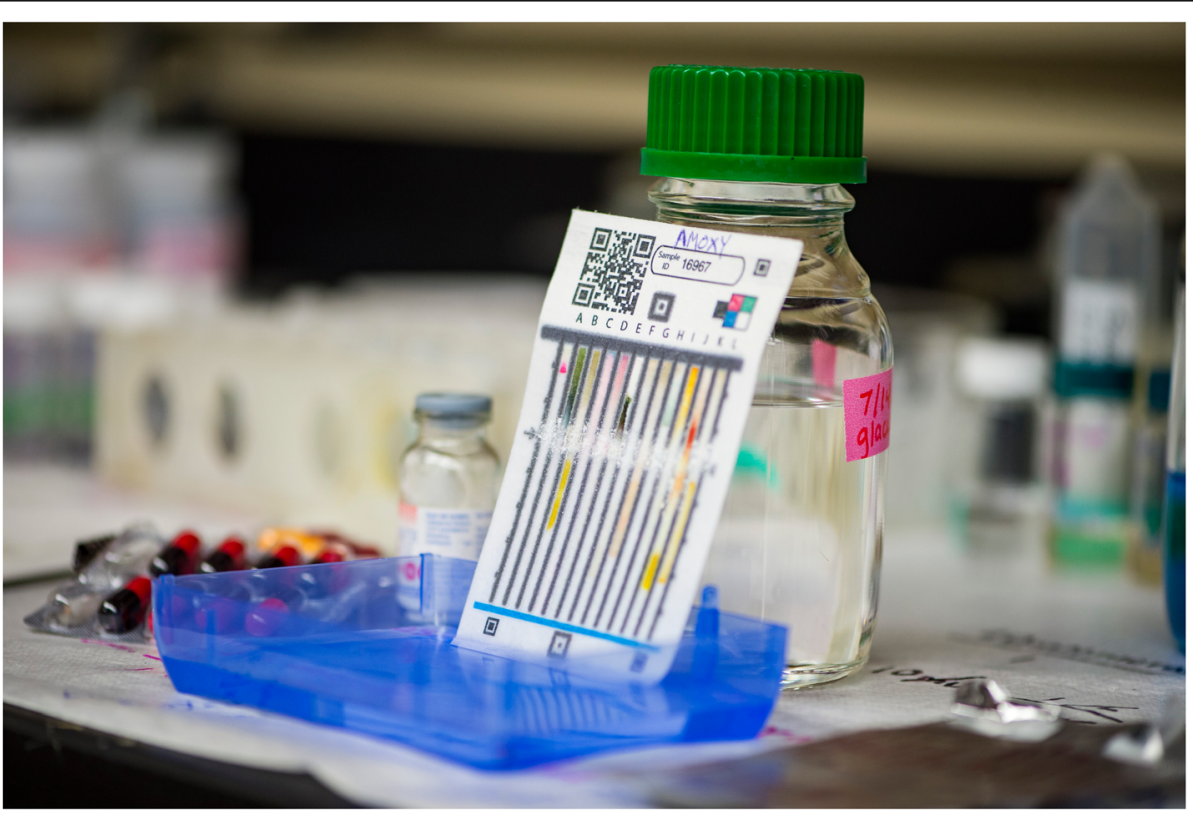

Fig. 4 Paper analytical device running a sample. Source: Barbara Johnson/University of Notre Dame, permission granted by Marya Lieberman, University of Notre Dame 


\section{PharmaChk: employing Chemiluminescence}

PharmaChK is an instrument being developed by researchers at Boston University. Although originally intended to perform field dissolution, it is now being modified to provide quantitative information on the drug substance of interest (Desai 2014; Ho et al. 2015). PharmaChk requires dissolving a sample in solution to measure its luminescence and is not yet commercially available.

\section{Speedy Breedy: employing Respirometry}

Speedy Breedy is a commercially available portable respirometer that measures pressure change in a vessel filled with a liquid sample to determine whether or not the sample has been contaminated with microbes. Although traditionally used for the analysis of foods, USP's technology review program, in collaboration with local partners, recently evaluated the instrument as a technology to screen the sterility of liquid samples (e.g., water for injection and artesunate injections). Speedy Breedy was evaluated in both laboratory and field settings in Ghana, India and Zimbabwe, respectively. The instrument was reliably able to detect bacterial contamination in liquid samples and less than 1 week was required to train staff to effectively use Speedy Breedy in field settings. Although this test is several days shorter than traditional sterility testing, with most protocols having $24 \mathrm{~h}$ run times or less, it does require continuous power for the entirety of the run (USP Technology Review: Speedy Breedy 2018).

\section{Screening technologies - needs, current efforts, and deployment strategies}

While information on capabilities of STs is available, a lot of the existing literature presumes a certain level of familiarity with the technology. There is a lack of guidance translating the analytical capabilities of screening technologies into effective field deployment and specifically within existing PMS systems.

Strategies and approaches for empowering prospective ST users in LMICs should focus on three objectives:

1. Develop guidelines and standards that describe how to evaluate and utilize STs

2. Disseminate comparable, rigorous, objective, and practical information about the strengths and limitations of STs

3. Provide user-specific guidance and support (including local capacity building) on ST deployment

\section{Guidelines and standards}

The absence of guidelines, public information, and standards that describe how to evaluate the suitability of STs contributes to the ineffective utilization of these technologies. Guidelines and standards would be particularly helpful in LMICs where the potential impact of STs is much greater than in high-income countries (Gostin and Buckley 2013; Pribluda et al. 2014). Access to guidelines and standards helps users ask the questions that enable them to determine the suitability of a given ST, such as whether an organization can afford the technology, whether the technology can screen medicines of interest, and how costly and difficult it is to train staff?

USP has published a proposed general chapter, < 1850> Evaluation of Screening Technologies, for Assessing Medicine Quality (USP 2018), which focuses on providing users with the guidelines and standards they need to address critical questions regarding ST utilization. The General Chapter was developed by a USP expert panel, comprised of professionals from regulatory agencies, the pharmaceutical industry, academia, and non-governmental organizations (USP 2018). Six ST applications are described as they relate to the analysis of pharmaceutical products, i.e. medicines, bulk drug substances, and excipients:

- Application I: Verification of packaging, labeling, origin, and appearance

- Application II: Major component ID

- Application III: Impuritiy ${ }^{1}$ ID

- Application IV: Major component quantification

- Application V: Impurity quantification

- Application VI: Performance characteristics (medicines only)

Table 2 builds on this work and lists the different STs discussed in this manuscript and specifies which types of applications are most appropriate for each ST and details the capital investment required to procure them.

(Information not obtained) - Instruments are commercially available, but pricing information could not be obtained.

(Not commercially available) - Instrument not commercially available, as a result, there is no pricing information available.

Future work should focus on the development of more detailed guidelines for the evaluation of all types of ST analyses. In addition, instrument manufacturers can leverage the proposed USP general chapter and future guidelines to improve their offerings. Incorporating PMS needs and perspectives in product design could also lead to more effective use of vibrational spectroscopy. For example, to better enable the use of vibrational spectroscopy for PMS, medicine manufacturers could consider minimizing the interferences, which impede these technologies. The use of colorants and opaque packaging could, for example, be reduced (Vickers et al. 2018). Although 
Table 2 Applications and capital costs of different screening technologies by technique or equipment

\begin{tabular}{|c|c|c|c|c|c|c|c|}
\hline \multirow[t]{2}{*}{ Screening Technology Techniques and Notable Equipment } & \multicolumn{6}{|c|}{ Application $^{a}$} & \multirow[t]{2}{*}{ Price Range $(U S D)^{b}$} \\
\hline & I & $\|$ & III & IV & V & $\mathrm{Vl}$ & \\
\hline Infrared spectroscopy & NA & + & NA & $t_{N}$ & NA & NA & $\$ 25,000-\$ 70,000$ \\
\hline Near infrared spectroscopy & NA & + & NA & $+_{N}$ & NA & NA & $\$ 7000-\$ 60,000$ \\
\hline Raman spectrometry & NA & + & NA & $t_{N}$ & NA & NA & $\$ 18,000-\$ 60,000$ \\
\hline X-ray diffraction spectroscopy & NA & $+_{\mathrm{L}}$ & NA & NA & NA & NA & information not obtained \\
\hline X-ray fluorescence spectroscopy & NA & + & $+_{L}$ & $+_{\mathrm{L}}$ & $+_{L}$ & NA & $\$ 30,000-\$ 60,000$ \\
\hline Ion mobility spectrometry & NA & + & + & $+_{\mathrm{L}}$ & $+_{L}$ & NA & $\$ 25,000-\$ 60,000$ \\
\hline CD5 - multi-spectral imaging & + & NA & NA & NA & NA & NA & Not commercially available \\
\hline Counterfeit Drug Identifier - multi-wavelength absorption & NA & + & NA & NA & NA & NA & $\$ 100$ but not commercially available \\
\hline GPHF Minilab ${ }^{T M}$ - chromatography and disintegration & NA & + & $+_{\mathrm{L}}$ & $+_{\mathrm{L}}$ & NA & + & $\$ 4500-\$ 10,000$ \\
\hline Paper analytical device - chromatography and wet chemistry & NA & + & NA & $t_{\mathrm{L}}$ & NA & NA & $\$ 1$ per test card \\
\hline PharmaChk - chemiluminescence & NA & NA & NA & $+_{N}$ & NA & NA & Not commercially available \\
\hline Speedy Breedy - respirometry (sterility) & NA & NA & $+_{\mathrm{L}}$ & NA & $+_{N}$ & NA & $\$ 4500-\$ 5000$ \\
\hline
\end{tabular}

(+) Intended application

$\left(+_{N}\right)$ Intended but non-routine application

$\left(+_{L}\right)$ Intended application with significant limitations e.g., able to analyze a limited number of drug substances

(NA) Not an intended application

${ }^{a}$ As per proposed USP General Chapter $<1850>$

${ }^{\mathrm{b}}$ Ranges reflect price quotes obtained from instrument vendors and information from the literature.

such product decisions are often necessary for ensuring product quality, these decisions can also be influenced by marketing strategies.

\section{Information}

Beyond the need for guidelines on how to evaluate STs is the need for comprehensive and objective information on the capabilities of STs. Several studies have evaluated one specific ST or compared two or three STs side-byside for specific medicines, mostly antimalarials (Batson et al. 2016; Green et al. 2015; Hajjou et al. 2013), but there are no detailed, standardized individual, or comparative evaluations of these STs for use in LMICs across multiple medicines. It remains unclear which STs are the most accurate, appropriate, and cost effective depending on the situation and how much training and preparation is realistically required for sustainable use (Fernandez et al. 2011; Hall et al. 2016; Kaur et al. 2016; Nayyar et al. 2015). For example, NRAs in LMICs do not have resources which describe how all STs available on the market work and why one may be more advantageous than another based on the country's circumstances. Anecdotally, program managers and laboratory staff from the Promoting the Quality of Medicines program have observed some NRAs in LMICs, if they have STs, are obliged to use what is selected or provided by a donor or device manufacturer. Kovacs et al. (2014) described the ability of STs in the field and laboratory to detect SF medicines, and listed each ST's need for electricity, sample preparation, reagents, portability, level of training required, and speed of analysis (Kovacs et al.
2014). In addition, all STs are grouped into three categories by cost: USD $\$ 10,000$, USD $\$ 10,000-100,000$, and greater than USD $\$ 100,000$. The paper identified two key issues when examining STs; (1) the unavailability of performance data on and (2) the absence of a reliable standard.

The Lao Oxford Mahosot Wellcome Trust Research Unit recently published a systematic review of established and emerging STs, highlighting available data and identifying gaps in the literature (Vickers et al. 2018), which included independent evaluations of STs with respect to their field readiness, ability to analyze different medicines, their cost effectiveness, their utility in different areas of the supply chain and their training requirements. The Lao Oxford Mahosot Wellcome Trust Research Unit has also undertaken a project, with funding from the Asian Development Bank, to perform a side-by-side laboratory and field evaluation as well as a cost effectiveness analysis of STs, eight of which are commercially available and three of which are in development (IDDO 2018). USP's Technology Review program is carrying out similar work to LOMWRU, evaluating emerging STs identified by experts. The first USP Technology Review evaluations were published in late 2017 and early 2018 with three more scheduled for early 2019. The results of the USP Technology Review evaluations have already informed ST procurement for regulatory authorities and the pharmaceutical manufacturers. The WHO Member State Mechanism also has a working group to survey the available STs (Substandard/spurious/falsely-labelled/ falsified/counterfeit 
medical products Report by the Director-General 2015). At the 6th meeting of the Member State Mechanism, both USP and the Lao Oxford Mahosot Wellcome Trust Research Unit, who are non-state members of the working group, presented on their various activities (WHO 2017a). This continued exchange of information and collaboration is important to avoid a duplication of efforts and leverage their respective expertise, resources, and processes. Furthermore, to leverage the momentum of these programs and other activities, resources need to be mobilized to target comparative evaluations of established and emerging STs, which have yet to be evaluated in any forum (Wilson et al. 2017).

\section{Deployment}

The user-specific deployment of STs requires thoughtfulness, adaptability, and a need to tailor approaches. First and foremost, understanding how, when, and where to use the various STs available is difficult; there is no panacea ST. There are pros and cons to using each technology. For example, challenges with successful implementation of vibrational spectroscopy instrumentation in LMICs stem from developing and maintaining adequate reference libraries and building reliable quantitative models for medicines (IDDO 2018; Vickers et al. 2018). A challenge in both field and laboratory testing is therefore determining how to combine tests and technologies for detecting SF medicines with maximum efficiency (Batson et al. 2016; Gostin and Buckley 2013; Roth et al. 2018a). To expect one ST to detect all SF medicines is unrealistic. The deployment of multiple STs is more often the rule than the exception when working to detect SF medicines. A handheld Raman spectrometer can determine if an incorrect drug substance is present in an artesunate injectable sample, while Speedy Breedy can determine it is not sterile.
Secondly, the effectiveness of STs is limited by the effectiveness of the overarching PMS system. Developing and maintaining a functional, sustainable, and risk-based PMS system is one of the core functions of any NRA (Wirtz et al. 2017). STs are an integral part of these systems but understanding where STs fit into this broader system and how their results should be used is complex and context specific. Answers to these questions depend on many factors, including the size of the NRA and country, the existing PMS system, the capacity of the National Quality Control Laboratory, and the local burden of disease.

Finally, the requirements of a ST vary by type of user. Table 3 compares some of the general parameters and their relative importance across different types of users. An important requirement for a retail pharmacist would be an ST that works through packaging to avoid destroying a medicine and forgoing the associated revenue. Contrastingly, an inspector analyzing bulk material is not concerned about packaging. An inspector's ST does, however, need to be able to identify the drug substance and potentially the proportion of excipients in a bulk mixture.

Any prospective ST user should perform a situational analysis to determine their specific requirements and determine the ST most appropriate for their quality challenge. To do this, ST users can work with domestic, regional, or international counterparts and partners who may have experience working in this field. Regional groups such as the African Medicine Quality Forum (NEPAD 2017) should promote dialogue and exchange information about best practices and experiences. Donors and other key stakeholders also need to mobilize resources to support the evaluation of emerging STs and to support the provision of technical assistance to those seeking to incorporate STs into their PMS systems.

Table 3 Screening technology parameter importance by user

\begin{tabular}{|c|c|c|c|c|c|c|c|c|c|c|}
\hline \multirow[t]{3}{*}{ User } & \multicolumn{10}{|l|}{ Parameter } \\
\hline & \multirow{2}{*}{$\begin{array}{l}\text { Non- } \\
\text { destructive }\end{array}$} & \multirow[t]{2}{*}{ Size } & \multirow[t]{2}{*}{ Rapid } & \multirow{2}{*}{$\begin{array}{l}\text { Ease } \\
\text { of } \\
\text { use }\end{array}$} & \multicolumn{6}{|c|}{ Application $^{a}$} \\
\hline & & & & & $\overline{1}$ & $\|$ & III & IV & V & $\overline{\mathrm{VI}}$ \\
\hline Consumer & I & 1 & V & V & V & - & - & - & - & - \\
\hline Customs & V & 1 & V & V & V & V & - & - & - & - \\
\hline NRA - Inspectorate & I & V & V & 1 & V & l & - & - & - & - \\
\hline NRA - National Quality Control Laboratory & - & - & 1 & - & V & V & V & V & V & V \\
\hline Pharmaceutical manufacturer & - & V & V & - & - & V & V & 1 & I & - \\
\hline Pharmacy & V & 1 & 1 & - & V & l & - & I & - & - \\
\hline Procurement agency & V & 1 & 1 & V & V & l & - & I & - & - \\
\hline Wholesaler / distributor & V & 1 & 1 & I & V & । & - & 1 & - & - \\
\hline
\end{tabular}

(I) Important

(V) Very important

(-) Relatively less important

${ }^{\mathrm{a}}$ Per proposed USP General Chapter Evaluation of Screening Technology for Assessing Medicine Quality 


\section{Conclusion}

The march of globalization creates ever increasing complexities in our ability to provide quality medicines as exemplified by the proliferation of SF medicines. SF medicine STs provide affordable, easy to use, and portable tools to address this global healthcare challenge and are revolutionizing the way SF medicines are detected in LMICs. Care must be taken, particularly with chemical analysis STs, to employ robust method development and validation processes. Many data analysis tools are available to leverage the information contained in spectroscopic data to attain the desired level of method performance. A variety of techniques and tailored equipment are also available to the ST analyst, the strengths and weaknesses of which must be considered. Development of public guidelines and standards will enable NRAs to evaluate the suitability of emerging STs. Technical support and local capacity building will expand and accelerate the utilization of STs and promote collaboration and work-sharing among stakeholders facing similar challenges. As STs continue to develop, they will increasingly enable LMICs to combat the preventable but enduring global public health crisis of SF medicines.

\section{Endnotes}

${ }^{1}$ Impurities refer to minor components of the medicine or drug substance and may be present due to product adulteration, contamination, or degradation. According to compendial methods, impurity specification levels are typically in the range of $0.1-1 \%$ of the nominal concentration.

\section{Abbreviations \\ FDA: Food and drug administration; ID: Identification; IMS: Ion mobility spectrometry; IR: Infrared; LMICs: Low- and middle- income countries; NIR: Near-infrared; NRA: National regulatory authority; PAD: Paper analytical device; PMS: Post-marketing surveillance; QC: Quality control; SF: Substandard and falsified; ST: Screening technology; TLC: Thin layer chromatography; USP: United States Pharmacopeia; V-ST: Vibrational spectroscopy screening technology; WHO: World Health Organization; XRD: X-ray diffraction; XRF: X- ray fluorescence}

\section{Acknowledgments}

We would like to thank Celine Caillet, Mick Deats, Richard Jaehnke, Diana Lee, Marya Lieberman, Nico Ranieri, Serena Vickers, and the members of the USP expert panel on the evaluation of screening technologies for assessing medicine quality for their expertise and feedback on various sections of the manuscript.

\section{Authors' contributions}

All authors conceptualized the manuscript. LR and KBB drafted and revised the manuscript. LR finalized the manuscript. DKB reviewed and provided input on the manuscript. All authors read and approved the final manuscript.

\section{Funding}

Not applicable.

\section{Availability of data and materials}

Not applicable.

\section{Competing interests}

The authors declare that they have no competing interests.
Received: 6 February 2019 Accepted: 11 June 2019

Published online: 08 July 2019

\section{References}

Bannerjee S, Sweet J, Lieberman M, Flynn P, Sweet C (2016) Visual recognition of paper analytical device images for detection of falsified pharmaceuticals. Paper presented at the IEEE winter conference on applications of computer vision, Lake Placid, NY, March 7-9

Batson JS, Bempong DK, Lukulay PH, Ranieri N, Satzger RD, Verbois L (2016) Assessment of the effectiveness of the CD3+ tool to detect counterfeit and substandard anti-malarials. Malar J 15:119

Bortoleto GG, Pataca LCM, Bueno MIMS (2005) A new application of X-ray scattering using principal component analysis - classification of vegetable oils. Anal Chim Acta 539:283-287 https://doi.org/10.1016/j.aca.2005.03.025

Bruker (2016) Introduction to X-ray fluorescence analysis (XRF) - fundamental principles, Instrumentation

Bruker (2017) XRF Data differences: quantitative, semi-quantitative, and qualitative data. https://www.bruker.com/products/x-ray-diffraction-and-elementalanalysis/handheld-xrf/xrf-data-primer-quantitative-semi-quantitativequalitative.html. Accessed 02/01/2018

Desai D (2014) Pharmachk: robust device for counterfeit and substandard medicines screening on developing regions

Fadeyi I, Lalani M, Mailk N, Van Wyk A, Kaur H (2015) Quality of the antibiotics--amoxicillin and co-trimoxazole from Ghana, Nigeria, and the United Kingdom. Am J Trop Med Hyg 92:87-94. https://doi.org/10.4269/ ajtmh.14-0539

Fernandez FM, Hostetler D, Powell K, Kaur H, Green MD, Mildenhall DC, Newton PN (2011) Poor quality drugs: grand challenges in high throughput detection, countrywide sampling, and forensics in developing countries. Analyst 136:3073-3082

Gostin LO, Buckley GJ (2013) Countering the problem of falsified and substandard drugs. National Academies Press, Washington, D.C.

GPHF (2017) Minilab factsheet

GPHF (2019) The GPHF-MInilab - Protection Against Counterfeit Medicines. https://www.gphf.org/en/minilab/. Accessed 20 Mar 2019

Green M (2018) Email exchange

Green MD, Hostetler DM, Nettey H, Swamidoss I, Ranieri N, Newton PN (2015) Integration of novel low-cost colorimetric, laser photometric, and visual fluorescent techniques for rapid identification of falsified medicines in resource-poor areas: application to Artemether-Lumefantrine. Am J Trop Med Hyg 92:8-16

Gryniewicz CM, Reepmeyer JC, Kauffman JF, Buhse LF (2009) Detection of undeclared erectile dysfunction drugs and analogues in dietary supplements by ion mobility spectrometry. J Pharm Biomed Anal 49:601-606 https://doi. org/10.1016/j.jpba.2008.12.002

Hajjou M, Qin Y, Bradby S, Bempong D, Lukulay P (2013) Assessment of the performance of a handheld Raman device for potential use as a screening tool in evaluating medicines quality. J Pharm Biomed Anal 74:47-55

Hall Z, Allan EL, van Schalkwyk DA, van Wyk A, Kaur H (2016) Degradation of artemisinin-based combination therapies under tropical conditions. Am J Trop Med Hyg. https://doi.org/10.4269/ajtmh.15-0665

Ho NT, Desai D, Zaman MH (2015) Rapid and specific drug quality testing assay for artemisinin and its derivatives using a luminescent reaction and novel microfluidic technology. Am J Trop Med Hyg 92:24-30. https://doi.org/10. 4269/ajtmh.14-0392

IDDO (2018) An evaluation of portable screening devices to assess medicines quality for national medicines regulatory authorities

Jaehnke R (2008) A concise quality control guide on essential drugs and other medicines. vol 2. Global Pharma Health Fund

Jaehnke R (2017) A concise quality control guide on essential drugs and other medicines: supplements 2010-2017 to volume II on thin layer chromatographc tests

Johnston A, Holt DW (2014) Substandard drugs: a potential crisis for public health. Br J Clin Pharmacol 78:218-243

Kaale E et al (2016) The quality of selected essential medicines sold in accredited drug dispensing outlets and pharmacies in Tanzania. PLoS One 11:e0165785. https://doi.org/10.1371/journal.pone.0165785

Kauffman JF, Westenberger BJ, Roberston JD, Guthrie J, Jacobs A, Cummins SK (2007) Lead in pharmaceutical products and dietary supplments. Regul Toxicol Pharmacol 48:128-134. https://doi.org/10.1016/j.yrtph.2007.03.001 
Kauffman JF et al. (2010) Pharmaceutical surveillance with rapid spetroscopic screening technologies American pharmaceutical Review

Kaur $\mathrm{H}$ et al (2016) Fake anti-malarials: start with the facts. Malar J 15:1

Khuluza F, Kigera S, Jahnke RW, Heide L (2016) Use of thin-layer chromatography to detect counterfeit sulfadoxine/pyrimethamine tablets with the wrong active ingredient in Malawi. Malar J 15:215. https://doi.org/10.1186/s12936016-1259-9

Kohli R, Mittal KL (2012) Developments in surface contamination and cleaning, volume 4 - detection, characterization, and analysis of contaminants. Elsevier, New York

Kovacs S, Hawes SE, Maley SN, Mosites E, Wong L, Stergachis A (2014) Technologies for Detecting Falsified and Substandard Drugs in low and middle-income countries. PLoS One 9. https://doi.org/10.1371/journal. pone.0090601

Lalani M et al (2015) Substandard antimalarials available in Afghanistan: a case for assessing the quality of drugs in resource poor settings. Am J Trop Med Hyg 92:51-58. https://doi.org/10.4269/ajtmh.14-0394

Lieberman M (2017) Email exchange

Martino R, Malet-Martino M, Gilard V, Balayssac S (2010) Counterfeit drugs: analytical techniques for their identification. Anal Bioanal Chem 398:77-92. https://doi.org/10.1007/s00216-010-3748-y

Maurin JK, Pluciński F, Mazurek AP, Fijałek Z (2007) The usefulness of simple X-ray powder diffraction analysis for counterfeit control—the Viagra ${ }^{\circledR}$ example. J Pharm Biomed Anal 43:1514-1518 https://doi.org/10.1016/j.jpba.2006.10.033

Milman BL (2011) Chemical identification and its quality assurance. Springer, Berlin

Nayyar GM et al (2015) Responding to the pandemic of falsified medicines. Am J Trop Med Hyg 92:113-118

Neospectra (2019) NIR spectroscopy - how it works. https://www.neospectra. com/nir-spectroscopy/. Accessed 31 Mar 2019

NEPAD (2017) African medicines quality forum to improve quality control of medicines

Newton P, Caillet C, Vickers S (2018) Email exchange

Nkansah P et al. (2017) Guidance for implementing risk-based post-marketing quality surveillance in low- and middle-income countries. U.S. Pharmacopeial Convention. The promoting the quality of Mediciens program, Rockville, Maryland

Ortiz RS et al (2012) Fingerprinting of sildenafil citrate and tadalafil tablets in pharmaceutical formulations via X-ray fluorescence (XRF) spectrometry. J Pharm Biomed Anal 58:7-11 https://doi.org/10.1016/j.jpba.2011.09.005

Petersen A, Held N, Heide L, on behalf of the Difäm EPNMSG (2017) Surveillance for falsified and substandard medicines in Africa and Asia by local organizations using the low-cost GPHF Minilab. PLoS One 12:e0184165. https://doi.org/10.1371/journal.pone.0184165

PQM (2017) Annual performance report 2017. Promoting the quality of medicines program,

Pribluda $V$ et al. (2014) The three-level approach: a framework for ensuring medicines quality in limited-resource countries pharmaceutical regulatory affairs 3

Ranieri N (2018) CDx details

Ranieri N et al (2014) Evaluation of a new handheld instrument for the detection of counterfeit artesunate by visual fluorescence comparison. Am J Trop Med Hyg 91:920-924. https://doi.org/10.4269/ajtmh.13-0644

Risha PG, Msuya Z, Clark M, Johnson K, Ndomondo-Sigonda M, Layloff T (2008) The use of minilabs to improve the testing capacity of regulatory authorities in resource limited settings: Tanzanian experience. Health Policy 87:217-222. https://doi.org/10.1016/j.healthpol.2007.12.010

Rodriguez J (2017) Ion mobility spectrometry. USP APEC Center of Excellence Pilot Program

Rodriguez J, Gryniewicz CM, Arzhantsev S, Kauffman JF, Buhse LF (2014) Rapid screening methods for pharmaceutical surveillance. Center for Drug Evaluation and Research, US Food and Drug Administration.

Roth L, Nalim A, Turesson B, Krech L (2018a) Global landscape assessment of screening technologies for medicine quality assurance: stakeholder perceptions and practices from ten countries BMC. Glob Health 14. https:// doi.org/10.1186/s12992-018-0360-y

Roth L et al (2018b) Expanding global access to essential medicines: investment priorities for sustainably strengthening medical product regulatory systems BMC. Glob Health 14. https://doi.org/10.1186/s12992-018-0421-2

Sabartova J, Toumi A, Policies DEMP, Health W (2011) Survey of the quality of selected antimalaria medicines circulating in six countries of sub-Saharan Africa. World Health Organization, Geneva
Safe, Secure, And controlled: managing the supply chain of antimicrobials (2015). The Review on Antimicrobial Resistance (AMR).

Shimadzu (2019) Raw materials identification testing by NIR spectroscopy and raman spectroscopy. https://www.shimadzu.com/an/ftir/support/tips/ letter20/raman.html. Accessed 31 Mar 2019

Substandard/spurious/falsely-labelled/ falsified/counterfeit medical products Report by the Director-General (2015). World Health Organziation, Geneva, Switzerland

USP (2018) < 1850> Evaluation of Screening Technologies for Assessing Medicine Quality, Rockville, U.S. Pharmacopeia

USP Technology Review: Speedy Breedy (2018). U.S. Pharmacopeia, Rockville, Maryland

Vickers S, Bernier M, Zambrzycki S, Fernandez FM, Newton PN, Caillet C (2018) Field detection devices for screening the quality of medicines: a systematic review. BMJ Glob Health 3. https://doi.org/10.1136/bmjgh-2018-000725

Weaver AA, Lieberman M (2015) Paper test cards for presumptive testing of very low quality antimalarial medications. Am J Trop Med Hyg 92:17-23. https:// doi.org/10.4269/ajtmh.14-0384

Weaver AA et al (2013) Paper analytical devices for fast field screening of Beta lactam antibiotics and Antituberculosis pharmaceuticals. Anal Chem 85:64536460. https://doi.org/10.1021/ac400989p

WHO (2013) Falsified lamivudine/zidovudine/nevirapine tablets: rapid identification using X-ray fluorescence technique WHO Drug Information, vol 27, p 5

WHO (2015) Substandard/spurious/falsely-labelled/falsified/counterfeit medical products - Report by the Director-General vol A68/33

WHO (2017a) Meeting of the Member State mechanism on substandard and falsified medical products

WHO (2017b) Report of the fifth meeting of the member state mechanism on substandard / spurious / falsely-labelled / falsified / counterfeit medical products. World Health Organization

WHO (2017c) A study on the public health and socioeconomic impact of substandard and falsified medical products. World Health Organization, Geneva

Wilson BK, Kaur H, Allan EL, Lozama A, Bell D (2017) A new handheld device for the detection of falsified medicines: demonstration on falsified artemisininbased therapies from the field. Am J Trop Med Hyg 96:1117-1123. https:// doi.org/10.4269/ajtmh.16-0904

Wirtz $V$ et al (2017) Essential medicine for universal health coverage the lancet commission, pp 403-476

Yu H, Le H, Lumetta S, Cunningham BT, Kaale E, Layloff T (2016) Smartphonebased thin layer chromatography for the discrimination of falsified medicines. In: 2016 IEEE SENSORS, Oct. 30 2016-Nov. 3 2016, pp 1-3. https:// doi.org/10.1109/ICSENS.2016.7808847

Zou W-B, Yin L-H, Jin S-H (2018) Advances in rapid drug detection technology. J Pharm Biomed Anal 147:81-88 https://doi.org/10.1016/j.jpba.2017.08.016

\section{Publisher's Note}

Springer Nature remains neutral with regard to jurisdictional claims in published maps and institutional affiliations.

\section{Submit your manuscript to a SpringerOpen ${ }^{\circ}$ journal and benefit from:}

- Convenient online submission

- Rigorous peer review

- Open access: articles freely available online

- High visibility within the field

- Retaining the copyright to your article

Submit your next manuscript at $>$ springeropen.com 